\title{
Extreme halophilic alcohol dehydrogenase mediated highly efficient syntheses of enantiopure aromatic alcohols
}

Diya Alsafadi, ${ }^{a^{*}}$ Safaa Alsalman ${ }^{\mathrm{a}}$ and Francesca Paradisi ${ }^{\mathrm{b}^{*}}$

Enzymatic synthesis of enantiopure aromatic secondary alcohols (including substituted, heteroaromatic and bicyclic structures) were carried out using the halophilic alcohol dehydrogenase ADH2 from Haloferax volcanii (HvADH2). This enzyme showed an unprecedented

substrate scope and absolute enatioselectivity. The cofactor NADPH was used catalytically and regenerated in-situ by the biocatalyst, in the presence of $5 \%$ ethanol. The efficiency of HVADH2 for conversion of aromatic ketones was markedly influenced by the steric and electronic factors as well as the solubility of ketones in the reaction medium. Furthermore, carbonyl stretching bands frequencies $\mathrm{v}(\mathrm{c}=\mathrm{o})$ have been measured for different ketones to understand the effect of electron withdrawing or donating properties of the ketones substituents on the reaction rate catalyzed by $H v A D H 2$. Good correlation was observed between $\mathrm{v}(\mathrm{c}=\mathrm{O})$ of methyl aryl-ketones and the reaction rate catalyzed by $\mathrm{HvADH} 2$. The enzyme catalyzed the reductions of ketone substrates on the preparative scale, demonstrating that HvADH2 would be a valuable biocatalyst for the preparation of chiral aromatic alcohols of pharmaceutical interest.

\section{Introduction}

Chirality is a key factor in the safety and efficacy of many drugs and thus the production of enantiopure drugs has become increasingly important in the pharmaceutical industry. ${ }^{1}$ Chiral aromatic secondary alcohols are widely used in synthetic organic and medicinal chemistry as key intermediates for the synthesis of various pharmaceutical products such as Zetia ${ }^{\circledR}$ (Ezetimibe), ${ }^{2}$ Prozac $^{\circledR}$ (Fluoxetine) ${ }^{3}$ and Emend $^{\circledR}$ (Aprepitant) $^{1}$ (Fig. 1).

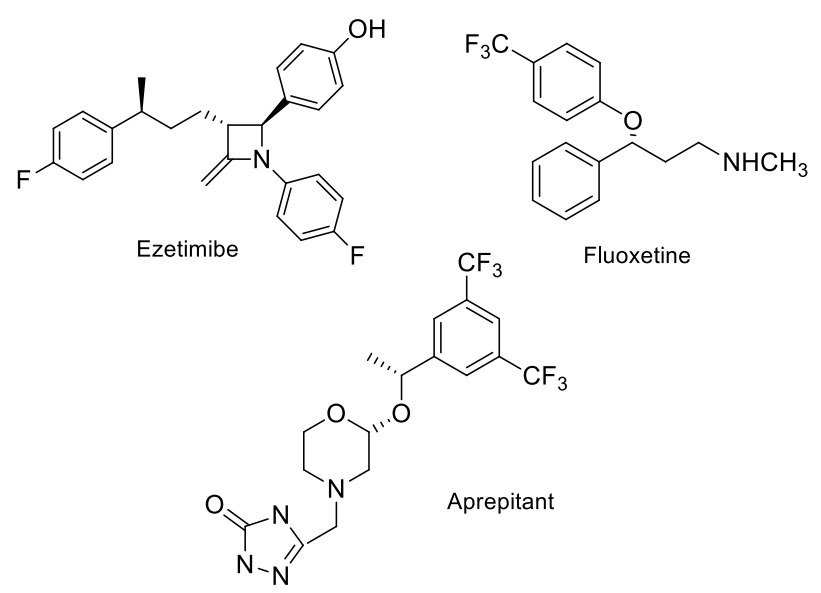

Fig. 1 Examples of drugs derived from chiral aromatic secondary alcohols precursors.

A variety of chiral metal complexes have been used as catalysts for enantioselective synthesis of chiral aromatic secondary alcohols ${ }^{4-5}$ however, biocatalytic transformation systems using cell-free enzymes ${ }^{6}$ or whole-cell microorganisms ${ }^{7}$ offers advantages with respect to high catalytic efficiency, mild reaction conditions, outstanding enantio-, regio- and chemoselectivity and being void of toxic metals. 
Alcohol dehydrogenases (ADHs, EC 1.1.1.1) are a class of nicotinamide adenine dinucleotide (phosphate) $\left[\mathrm{NAD}(\mathrm{P})^{+}\right]$-dependent enzymes that catalyse the reversible reduction of aldehydes and ketones to their corresponding alcohols. ${ }^{8}$ Asymmetric reduction of the prochiral ketones using ADH is an important tool for industrial production of enantiopure alcohols. ${ }^{9-10}$ Additionally, ADHs can accomplish dynamic kinetic resolution, ${ }^{11-12}$ and deracemization of racemic alcohols ${ }^{13-14}$ as well as racemization of enantiopure alcohols, ${ }^{15-16}$ processes by which high yields (theoretically up to $100 \%$ ) of a single enantiomer can be obtained.

Although several ADHs have been identified from various organisms, their substrate scope tends to be limited and mutagenesis has often been necessary to expand the range of substrates. ${ }^{17,18}$ Furthermore, cofactor regeneration must be taken into consideration to minimize the economic impact of the biocatalytic process. As a consequence, several methods have been developed to regenerate the cofactor in ADH-catalyzed reactions such as a coupled enzyme system, or with the use of a second substrate. ${ }^{6}$

Steric and electronic properties of substituent groups also play a major role on the biocatalytic reduction of aromatic ketones. ADHs from Pichia glucozyma, ${ }^{19}$ Aromatoleum aromaticum ${ }^{20}$ and Lactobacillus brevis $^{21}$ have been applied in the asymmetric reduction of acetophenone derivatives with different size and electronic properties. The formations of aromatic secondary alcohols were explained by substrate docking experiment and interaction energy values, ${ }^{20} \sigma$-Hammett coefficients ${ }^{19}$ and IR carbonyl stretching bands. ${ }^{21}$ The results have provided valuable insight into the understanding of how ADHs behave with aromatic ketones.

There are a limited number of ADHs effectively useful in the synthesis of chiral aromatic secondary alcohols which have been described in the literature. For instance, alcohol dehydrogenase from Ralstonia sp. (RasADH) catalyzed the reduction of aromatic ketones containing bulky substituents with high enantioselectivity. ${ }^{22}$ This type of ADHs has great relevance in asymmetric synthesis because of its broad substrate scope. ADH from Thermus thermophilus $\mathrm{HB} 27^{23}$ was used in the asymmetric reduction of aromatic ketones with anti-Prelog selectivity. Considering the value of aromatic chiral alcohols, it will be increasingly important to identify more ADHs that can be employed in such processes with high enantioselectivity. Extremophiles represent a promising source of robust biocatalysts with industrial applications. A number of ADHs from extremophiles have been identified and studied with respect to their ability to work in harsh industrial conditions such as high temperature ${ }^{24}$ and non-aqueous medium. ${ }^{25-}$ 26

As part of our effort to develop effective ADHs for synthesis of industrially valuable chiral alcohols, we developed and characterized four novel alcohol dehydrogenases from halophilic bacteria. ${ }^{27-29}$ In particular; alcohol dehydrogenase from the extreme halophile Haloferax volcanii (HvADH2) showed remarkable activity and stability in the presence of different organic solvents. ${ }^{27}$ For easy recovery from the reaction mixture and reutilization, $\mathrm{HVADH} 2$ was also seccesfully immobilized on solid support. ${ }^{30}$ The initial substrate screening for this enzyme showed encouraging results towards benzyl alcohol ${ }^{27}$ which prompted further in depth investigation of $\mathrm{HVADH} 2$ with industrially relevant compounds.

Herein, we report on the application of $H V A D H 2$ for the synthesis of a very broad range of aromatic secondary alcohols. The versatility of this enzyme and its adaptability to optimized reaction conditions is uncommon in the examples found to date in the literature. The effect of the steric, electronic and solubility properties of the substrates on the reaction catalyzed by HVADH2 is also discussed. Finally, IR absorption bands of the carbonyl group are measured and correlated with the reaction rate catalyzed by HvADH2. 


\section{Results and discussion}

\section{Reduction of aromatic ketone using HVADH2}

Aromatic secondary alcohols such as 1-phenylethanol, 1-phenyl-2-propanol and 4-phenyl-2-butanol were investigated as possible substrates for the alcohol dehydrogenase enzyme from the extreme halophile Haloferax volcanii (HvADH2) in the oxidation reaction. Preliminary activity assays suggested that HvADH2 accepted aromatic alcohol substrates with most marked activity observed against 1-phenyl-2-propanol. Therefore, 1-phenyl-2-propanone was used as the model substrate in order to optimize the reaction conditions $(\mathrm{pH}$, temperature, organic solvents, and co-factor recycling system) in the reductive direction with HvADH2 (Fig 2).

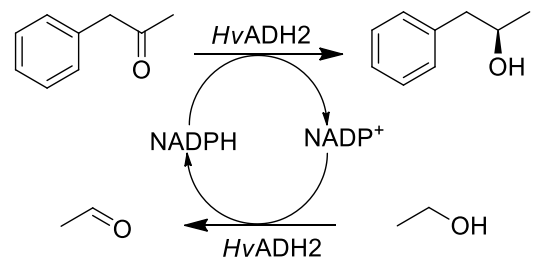

Fig. 2 Reduction of 1-phenyl-2-propanone using HvADH2.

Initially, the effect of $\mathrm{pH}$ on the conversion of 1-phenyl-2-propanone was investigated at $\mathrm{pH} 6$ and $\mathrm{pH} 8$, where $\mathrm{HVADH} 2$ exhibited the highest activity for the reductive reaction. ${ }^{27}$ The reactions were performed either utilizing a substrate-coupled approach ( $5 \%$ ethanol) to regenerate the co-factor or with an excess of NADPH. In both cases, the best conversion was observed at $\mathrm{pH} 8$, in fact no reaction was observed at $\mathrm{pH} 6$ in the presence of 5\% ethanol and catalytic amount of NADPH likely due to a stability issue at the lower $\mathrm{pH}$ over an extended period of time.

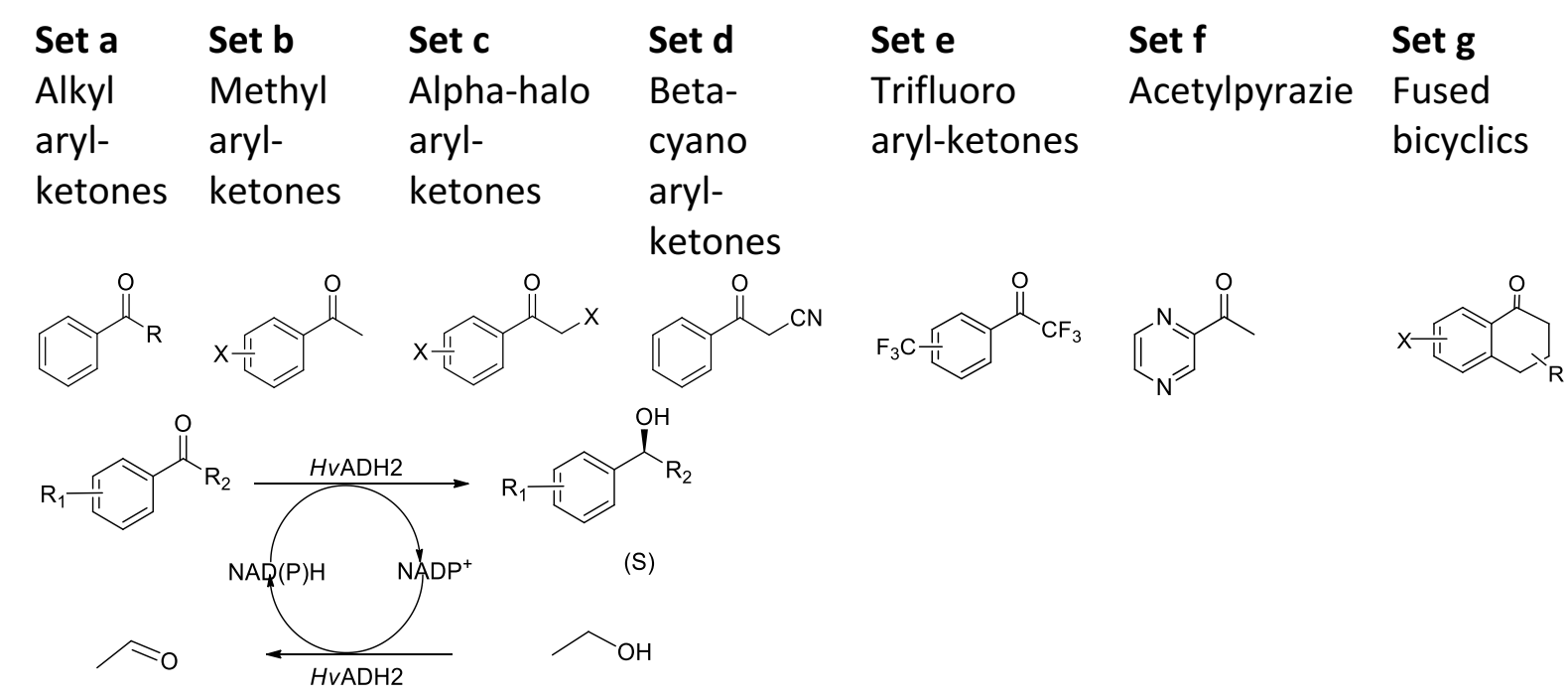

Fig. 3 Various aromatic ketones derivatives and general scheme of their reduction with HvADH2.

For the cofactor regeneration, alcohols that had been used as substrates in the oxidative reactions were tested in 5\% concentrations, including 2-propanol, 1-propanol and 1-butanol, ${ }^{27}$ however only ethanol 
performed well. To determine the optimum concentration of ethanol as co-substrate in the reaction, different concentrations of ethanol (5\%-20\%) were trialled but already at $10 \%$ the conversion dropped to $60 \%$ with respect to the lower amount (results not shown). Likely acetaldehyde accumulating in the media is toxic to the enzyme and $5 \%$ ethanol was chosen for all subsequent reactions.

To investigate the influence of organic solvents and temperature, the reduction of 1-phenyl-2-propanone was carried out in the presence of $5 \%$ of organic solvents (dimethyl sulfoxide, methanol and acetonitrile) at $25^{\circ} \mathrm{C}$ and $50^{\circ} \mathrm{C}$. The reactions were monitored by HPLC analysis as described in the experimental section. The maximum conversion of 1-phenyl-2-propanone (95\%) was observed in the presence of $5 \%$ of acetonitrile at $25^{\circ} \mathrm{C}$, while the conversion recorded in DMSO and methanol was lower (62 \% and $37 \%$, respectively) at the same temperature.

\section{Substrate scope and stereoselectivity of HvADH2}

Several aromatic ketones falling into seven structural classes were selected based on their potential for the production of industrially valuable chiral aromatic alcohols. Wild-type HvADH2 was used for the enantioselective reduction of each substrate class (Fig. 3).

The reactions were monitored by HPLC and the purity of the product determined by ${ }^{1} \mathrm{H}-\mathrm{NMR}$ analysis. The percent conversion of ketones and the e.e. values of the produced alcohols are reported in Table 1.

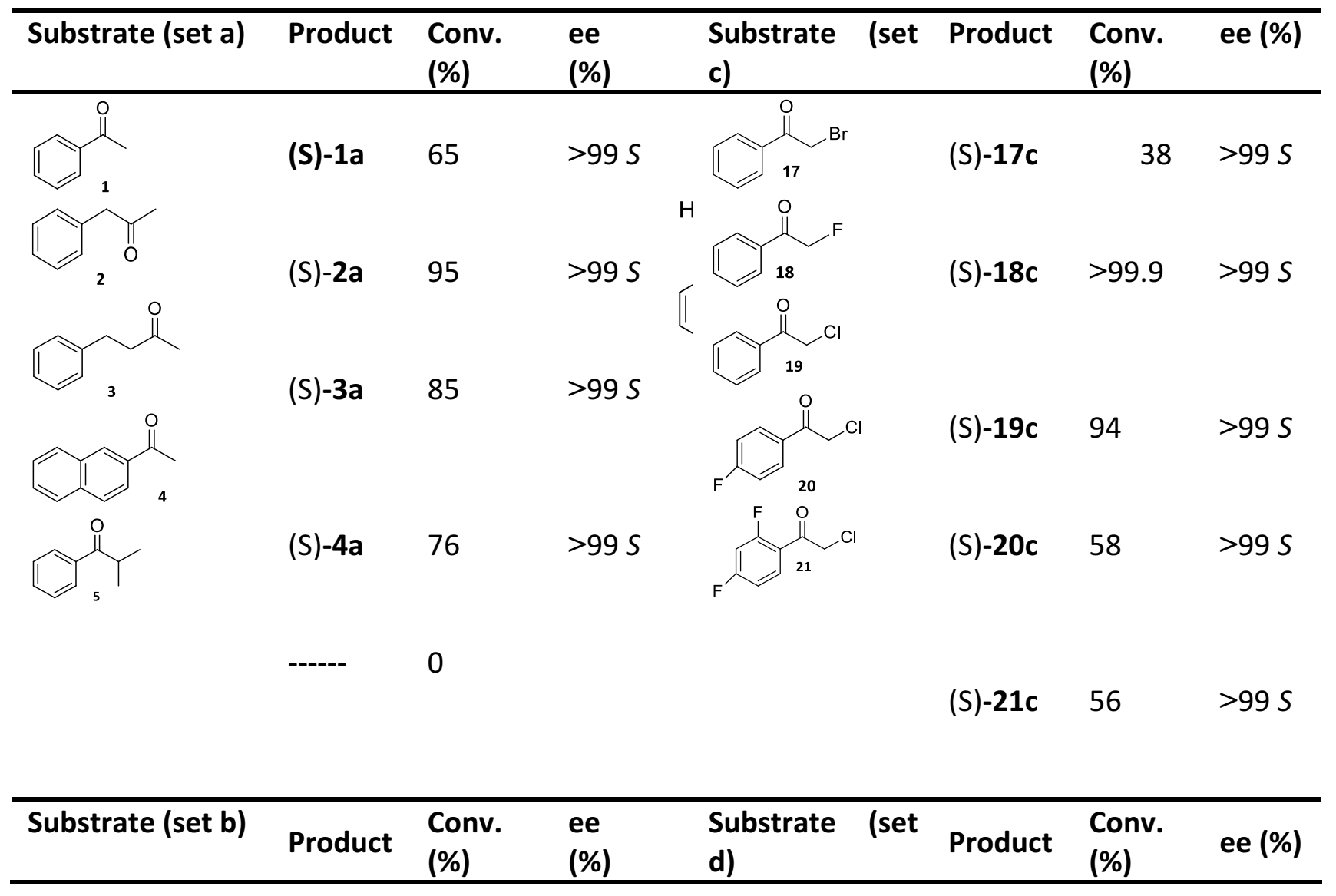




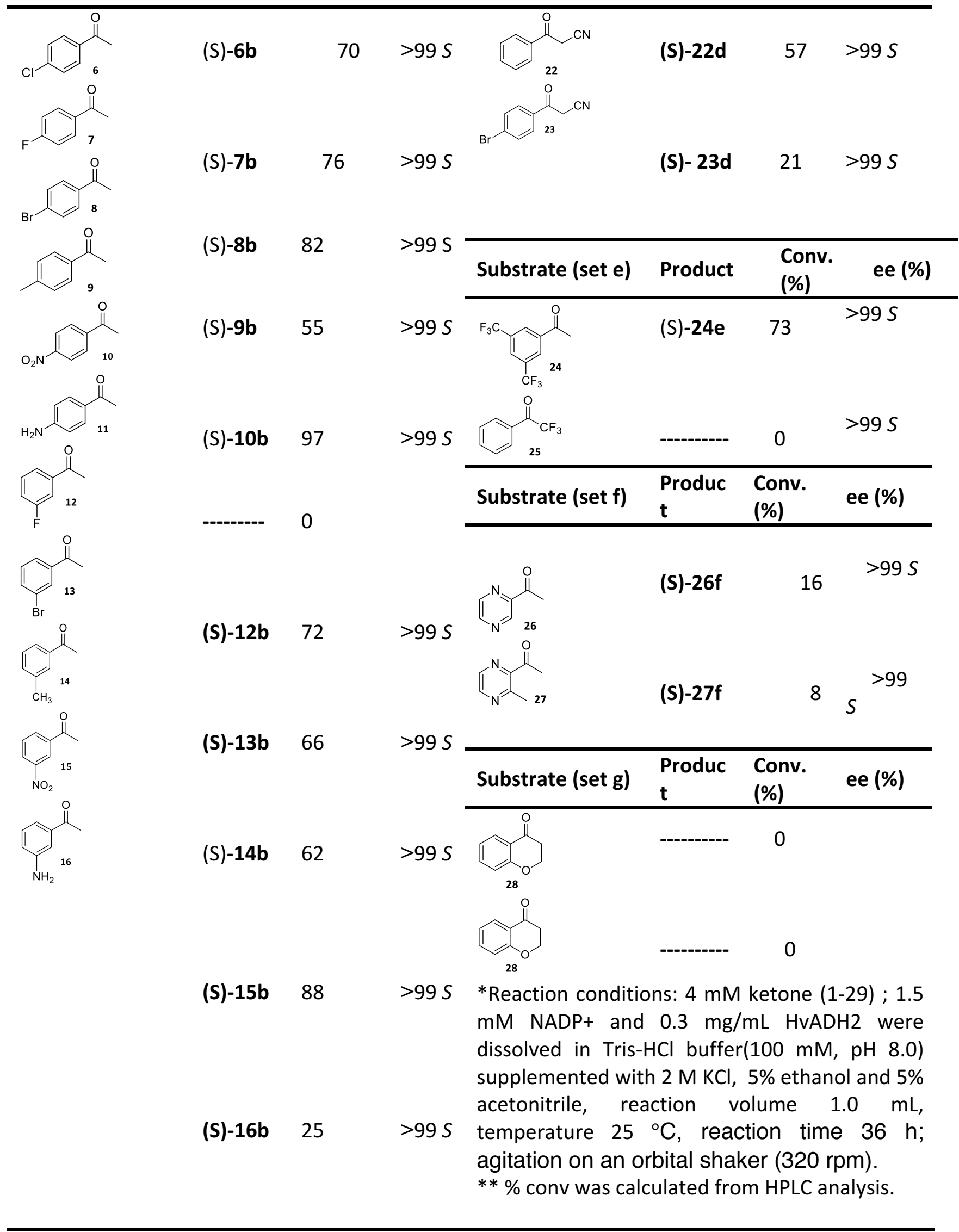

Table 1 Results from the reduction of various aromatic ketones derivatives using $\mathrm{HvADH} 2$ 
HVADH2 follows rigorously Prelog's rule yielding exclusively $(S)$-alcohols ( $100 \%$ e.e.) independently of the substrate tested. Other dehydrogenases/reductases reported in the literature show mixed selectivity depending on the substrate. For example, (S)-1phenylethanol dehydrogenase from Aromatoleum aromaticum showed strong (S)enantioselectivity however, a racemic mixture of 1-(4-aminophenyl)ethanol was observed when $4^{\prime}$ - aminoacetophenone 11 was tested. ${ }^{20}$ Additionally, acetophenone 1 was reduced to $(R)$-1-phenylethanol (opposite enantiomer) with $95 \%$ e.e using benzilketoreductase KRED1-Pglu isolated from Pichia glucozyma. ${ }^{19}$ Alpha-halo aryl-ketones such as phenacyl bromide 17 and phenacyl chloride 19 were reduced to their corresponding $(S)$-alcohols using the short-chain dehydrogenase from Streptomyces griseus while, methyl aryl-ketones such as 4'-chloroacetophenone 6 and 4-bromoacetophenone 8 were reduced to $(R)$-alcohols using the same biocatalyst. ${ }^{31}$ Interestingly, the stereoselectivity of $\mathrm{HVADH} 2$ was maintained with all aromatic ketones (1-27) tested in this work.

Clearly, the efficiency of HVADH2 for conversion of alkyl aryl-ketones (set a) were different and markedly influenced by steric factors such as substrate size and the type of alkyl chain, linear or branched. For liner alkyl chain ketones (1-4), the smallest substrate acetophenone 1 showed the lowest conversion (65\%). On the other hand, HvADH2 effectively catalyzed the enantioselective reduction of alkyl aryl-ketones as the alkyl chain between the carbonyl group and phenyl group became longer $\mathbf{2}$ and 3. Notably, good conversion (76\%) was observed with the bulkier substrate 4 . Thus, bulky groups might be better stabilized in the enzyme active site in such a way to increase the catalytic efficiency of the enzyme. In line with our initial substrate scope investigation, ${ }^{27}$ no conversion was observed with the substrate containing a branched group 5. It is worth noting that the influence of branched alkyl chain on the ketones reduction by $A D H$ was not observed before in the literatures.

The catalytic activity of HvADH2 on methyl aryl-ketones substrates (set b) was investigated using different meta- and para-substituted of acetophenone derivatives (6-16) and compared to acetophenone $\mathbf{1}$ (Table 1).

HVADH2 effectively catalyses the reduction of para-halogenated acetophenone derivatives (6-8), with up to $80 \%$ conversion. Para-methylacetophenone 9 gave satisfactory conversion (55\%) and the best conversion was reported with para-nitroacetophenone 10 which yielded the corresponding $(S)$-alcohol almost quantitatively. In contrast, para-amionoacetophenone 11 was not reduced by the enzyme, likely due to the strongly electron donating properties of the amino group as also noted by Contente et al. ${ }^{19}$ The difference on the catalytic activity of $H v A D H 2$ toward acetophenone derivatives could be explained by the electronic effect of the substituents on the reactivity of the carbonyl group. A similar trend was reported on an ADH from $L$. Brevis ${ }^{21}$ and a benzyl reductase from P. glucozyma. ${ }^{19}$ However, for other ADHs, the electronic properties of substituted acetophenones were not as significant. ${ }^{32}$

A more comprehensive study of the influence of the meta-position on the HvADH2 catalytic activity was carried out. In general, acetophenone derivatives with halogenated substituents at meta-position (12 and $\mathbf{1 3}$ ) represent suitable substrates, too. However, the halogenated meta-substituted acetophenones gave less satisfactory results compared with their parasubstituted counterpart. This behavior was not observed with methyl, nitro and amino acetophenone substituents (14-16), which gave better conversion rate at meta-position than para-position. The influence of acetophenone substituents on the reaction rate catalyzed by $\mathrm{HVADH} 2$ was further investigated by monitoring the carbonyl stretching bands with IR studies (see below). 
For the alpha-halo aryl-ketones (17-21), it was noticeable that the conversion rate for afluorinated ketone 18 with $H V A D H 2$ was better than a-chlorinated ketone 19 and this one

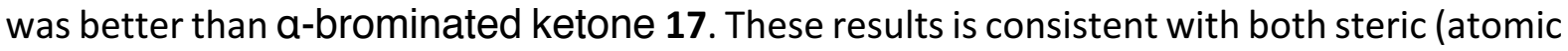
size $\mathrm{F}<\mathrm{Cl}<\mathrm{Br}$ ) and electronic (high electronegative $\mathrm{F}$ atom at a-position) factors of the groups.

Interestingly, phenacyl chloride 19 was converted faster than 2-chloro-4'fluoroacetophenone 20 and 2-chloro-2',4'-difluoro-acetophenone 21 with HVADH2. This indicated that the substituents on the phenyl ring of a-halo-ketone adversely affected the rate of enzymatic reaction. Clearly, for substrates $\mathbf{2 0}$ and $\mathbf{2 1}$ the steric effect is predominant in influencing the reaction rate rather than the electronic effect. This observation is also confirmed from the conversion value of 4 -fluoroacetophenone 7 (76\%) which was higher than 20 and 21 substrates.

The choice of solvent played a major role in the enzymatic reaction of $\mathrm{HvADH} 2$ with betacyano aryl-ketones (set $d$ ). The reduction of benzoylacetonitrile 22 and 4bromobenzoylacetonitrile $\mathbf{2 3}$, did not proceed when the reaction was performed in an aqueous buffer containing acetonitrile (optimum co-solvent). Therefore, acetonitrile was replaced by dimethyl sulfoxide in an effort to increase the solubility of substrates containing polar cyano group at a-position. As expected, the conversion yields for the substrates $\mathbf{2 2}$ and $\mathbf{2 3}$ were greatly improved with the addition of the high polar solvent (DMSO) into the reaction buffer.

Enantiopure $1 R$-[3,5-bis(trifluoromethyl)phenyl] ethanol and its $(S)$-enantiomer are key chiral intermediates in the synthesis of Aprepitant (a NK-1 receptor antagonist). ${ }^{33}$ The reduction of 24 had been previously reported using different microbial strains with varying degree of success. ${ }^{34}$ Here, 24 e was successfully obtained with $\mathrm{HvADH} 2$ in $73 \%$ yield and $100 \%$ e.e.

2,2,2-trifluoroacetophenone $\mathbf{2 5}$ was tested as substrate for HvADH2. Surprisingly, no conversion was observed with this substrate. Acetylpyrazine compounds $\mathbf{2 6}$ and $\mathbf{2 7}$ were only minimally active affording low conversions (<20\%). $\mathrm{HvADH} 2$ like many $\mathrm{ADHs}^{35}$ fails in the reduction of fused cyclic ketones such as $\mathbf{2 8}$ and $\mathbf{2 9}$ which are particularly challenging.

\section{IR carbonyl stretching bands study}

To understand the electronic effect of aromatic ketones substituents on the reaction rate catalyzed by $\mathrm{HvADH} 2$, the carbonyl stretching bands for different ketones were measured and plotted against the \% conversion of aromatic ketones. The frequency values of the carbonyl bands can be used to predict the carbonyl bond character in the presence of electron withdrawing group (EWDG) or electron donating group (EDG). Ketones containing EWG expected to absorb at low frequency values (low double-bond character), whereas EDG suppress the delocalization of the aryl-conjugated carbonyl and the carbonyl stretching bands observe at high frequency.

Good correlation was observed between the IR carbonyl absorption bands of set $b$ ketone substrates 6-16 and the reaction rate catalyzed by $\mathrm{HVADH} 2$ (Fig. 4). Unexpectedly, the IR absorption frequencies of the carbonyl group increased with the reaction rate. Therefore, ketones containing EWG absorb at higher frequencies (higher double-bond character), which is not in line with the evidence of increased electrophilicity of the carbonyl carbon by EWG. A similar trend has been previously reported with the nucleophilic acyl substitutions of different esters $^{36}$ and the reduction of ketones using ADH from Lactobacillus brevis. ${ }^{21}$ The authors attributed the high reactivity of esters/ketones to the decrease of the esters/ketones ground 
state resonance stabilization and this appears to be due to effect of EWG. In contrast, no correlation was observed between IR carbonyl absorption bands of ketone substrates from sets ( $a, c, d, e$ and $f$ ) and the reaction rate catalyzed by HVADH2 (data not shown). This could be attributed to the influence of other factors on the reaction rate such as the substrate solubility and substrate size.

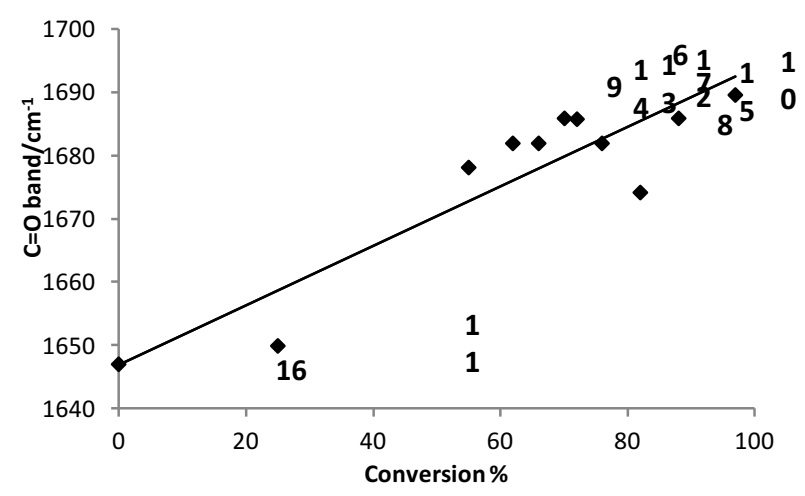

Fig. 4 Correlation between IR carbonyl stretching bands of substrates 6-16 and the \% conversion of aromatic ketones in the reaction catalyzed by $\mathrm{HvADH} 2$. The correlation coefficient $r^{2}=0.83$.

\section{Conclusion}

There is considerable interest in alcohol dehydrogenases as biocatalysts in the pharmaceutical industry for their ability to produce valuable chiral alcohols. In this work, we have shown that alcohol dehydrogenase from the halophilic archaeon Haloferax volcanii (HVADH2) effectively catalyzes the enantioselective reduction of a large selection of aromatic ketones with high enantiomeric purity to give (S)-form of aromatic secondary alcohols. The co-factor NADPH can be recycled by co-substrate addition ( $5 \%$ ethanol).

The conversion of aromatic ketones was different and influenced by (i) steric factors, (ii) electronic factors, and (iii) ketone solubility. No standard correlation parameters could be used to explain the conversion of aromatic ketones. For example, the conversion of alkyl arylketones (set a) were influenced predominantly by sterics however, the conversion of alphahalo aryl-ketones (set c), was influenced by both steric and electronic factors. Additionally, the reduction of cyano aryl-ketones (set d) was greatly dependant on solubility of ketones in the reaction medium.

IR absorption band of the carbonyl group was then used to investigate the effect of aromatic ketones substituents on the reaction rate catalyzed by $\mathrm{HvADH} 2$ and it was concluded that electron withdrawing groups have positive effect on ketones reactivity by destabilizing its ground-state resonance.

The results confirmed the suitability of $\mathrm{HVADH} 2$ as a valuable biocatalyst for the preparation of chiral secondary alcohols of pharmaceutical interest.

\section{Experimental}

Chemicals

All reagents and solvents, unless stated otherwise, were purchased as analytical grade.

Substrates, reagents, and cofactors were purchased either from Sigma Aldrich or Apollo Scientific Ltd, UK. Racemic alcohols were prepared from the corresponding ketones as 
follows. Ketones 1-29 (15 mmol) were dissolved in methanol $(2 \mathrm{~mL})$ at $0^{\circ} \mathrm{C}$. Sodium borohydride ( 0.5 equiv) was added and the reaction mixture was incubated with shaking for $1 \mathrm{~h}$ at $0^{\circ} \mathrm{C} .1 \mathrm{M} \mathrm{HCl}$ was added dropwise to maintain the reaction temperature below 5 ${ }^{\circ} \mathrm{C}$. Methanol was evaporated using a gentle stream of nitrogen. Then, water $(5 \mathrm{~mL})$ was added and the solution was extracted with ethyl acetate $(3 \times 5 \mathrm{~mL})$. The organic layers were combined and dried over $\mathrm{Na}_{2} \mathrm{SO}_{4}$. The solvent was evaporated and the residue was subjected to chromatography separation.

\section{Protein expression and purification}

HVADH2 was homologously overexpressed, using the vector pTA963 and host strain $H f x$. volcanii. The vector features an inducible L-tryptophan promoter and has a hexahistidine-tag, which allowed for easy purification by immobilized metal affinity chromatography (IMAC). ${ }^{27}$ Haloferax volcanii was grown overnight at $45{ }^{\circ} \mathrm{C}$ on $270 \mathrm{~mL}$ Hv-YPC medium. Protein overexpression was induced by the addition of $30 \mathrm{~mL}(50 \mathrm{mM})$ L-tryptophan to final volume $300 \mathrm{~mL}$ of growth medium. After $24 \mathrm{~h}$ the culture was harvested by centrifugation at 4,000 rpm for $10 \mathrm{~min}$. Cell pellets were resuspended in lysis buffer $(50 \mathrm{mM}$ Tris- $\mathrm{HCl}, \mathrm{pH} 8$, containing $\mathrm{KCl}(3 \mathrm{M})$ and disodium EDTA $(2 \mathrm{mM})$ ). Cells were lysed by sonication until the suspension was no longer turbid. The cell lysate was clarified by centrifugation at $18,000 \mathrm{rpm}, 4^{\circ} \mathrm{C}$ for $30 \mathrm{~min}$. After centrifugation, the supernatant was filtered through a $0.45 \mu \mathrm{m}$ membrane and loaded on to a chromatography column filled with Ni Sepharose 6 Fast Flow resin. The Histagged protein eluted when $10 \%$ ( $5 \mathrm{mM}$ disodium EDTA) to $30 \%$ (15 mM disodium EDTA) of elution buffer was applied. The purity of protein samples was assessed by sodium dodecyl sulfate polyacrylamide gel electrophoresis (SDS-PAGE).

\section{Analytical methods}

The activity of $H V A D H 2$ was analyzed spectrophotometrically by detecting the increase in absorbance at $340 \mathrm{~nm}$, which corresponds to the formation of the cofactor NADPH, using a Biochrom Libra S50 UV-Visible spectrophotometer. Enantiomeric excess (e.e.) and conversion rate were determined by normal phase high performance liquid chromatography (HPLC) using an Agilent 1100 series HPLC with chiral columns as the following: Chiralcel OJ-H (250x4.6 mm and $5 \mu \mathrm{m}$ silica gel), Dr. MAISCH Germany, Reprosil Chiral-BM (250x4.6 $\mathrm{mm}$ and $5 \mu \mathrm{m}$ silica gel) alternative to OB-H column and Phenomenex USA, Lux cellulose-1 column ( $250 \times 4.6 \mathrm{~mm}$ and $10 \mu \mathrm{m}$ silica gel) alternative to OD-H column. ${ }^{1} \mathrm{H}-\mathrm{NMR}$ spectra were recorded with Bruker $500 \mathrm{MHz}-$ Avance III spectrometer. Chemical shifts $(\delta)$ are expressed in ppm and coupling constants $(J)$ are expressed in $\mathrm{Hz}$. Optical rotation values $\left([\mathrm{a}]^{20}{ }_{\mathrm{D}}\right)$ were measured on a PolAAr 21 polarimeter at $589 \mathrm{~nm}$ in a $1 \mathrm{dm}$ cuvette at $20^{\circ} \mathrm{C}$. Fourier transform infrared (FT-IR) spectra for ketones were obtained in the range $400 \mathrm{~cm}^{-1}$ to $4,000 \mathrm{~cm}^{-1}$ using IR-Prestige-21 Shimadzu FT-IR spectrophotometer.

\section{General procedure for reduction of ketones and sample pre-treatment for HPLC analysis}

Ketone reduction was carried out in $5 \mathrm{~mL}$ screw-capped test tubes under the following conditions: $4 \mathrm{mM}$ ketone (1-29), $1.5 \mathrm{mM} \mathrm{NADP}^{+}$and $0.3 \mathrm{mg} / \mathrm{mL} H v A D H 2$ were dissolved/ suspended in $1 \mathrm{~mL}$ of $100 \mathrm{mM}$ Tris $-\mathrm{HCl} \mathrm{pH} 8.0$ supplemented with $2 \mathrm{M} \mathrm{KCl}, 5 \%$ ethanol and $5 \%$ acetonitrile. For benzoylacetonitrile 22 and 4-bromobenzoylacetonitrile 23, 5\% DMSO 
was used as co-solvent instead of acetonitrile. All reactions were incubated in an orbital shaker at $25^{\circ} \mathrm{C}, 320 \mathrm{rpm}$ for $96 \mathrm{~h}$.

The reaction mixtures were extracted with ethyl acetate $(3 \times 500 \mu \mathrm{L})$. The organic layer was dried over anhydrous sodium sulfate and transferred to a HPLC vial. The ethyl acetate was evaporated using a gentle stream of nitrogen and the residue was re-dissolved in $1 \mathrm{~mL}$ hexane. The solubility of 4-aminoacetophenone 11, 3-aminoacetophenone 16 and 2acetylpyrazine $\mathbf{2 6}$ in hexane was low and isopropanol was used in this case.

\section{General procedure for reduction of ketones on quantitative scale}

The biotransformation on the preparative scale was set up on a $20 \mathrm{mM}$ for each substrate. The reactions were performed as described before on the analytical scale. The alcohols from set $a, b$ and $c$ were prepared with $H v A D H 2$ in moderate to very good yields (30-90\%). The isolated yields for alcohols from set $f$ and $d$ were low (20\%). To fully characterize the alcohols the products residue were purified by preparative TLC using n-hexane/ethyl acetate $(3: 1, v / v)$ as an eluent Full spectra and analysis are reported in the ESI.

\section{Acknowledgments}

This research study was financially supported by the Scientific Research Support Fund (project No. Bas/1/01/2014).

\section{References}

1 R. N. Patel, Biomolecules, 2013, 3, 741-777.

2 A. S. Rowan, T. S. Moody, R. M. Howard, T. J. Underwood, I. R. Miskelly, Y. He and B. Wang, Tetrahedron: Asymmetry, 2013, 24, 1369-1381

3 D. Zhu, C. Mukherjee and L. Hua, Tetrahedron: Asymmetry, 2005, 16, 3775-3278

4 A. J. Blake, A. Cunningham, A. Ford, S. J. Teat and S. Woodward, Chem. Eur. J., 2000, 6, 3586-3594.

5 T. Reetz and X. G. Li, J. Am. Chem. Soc., 2006, 128, 1044-1045.

6 Y. Inoue, Y. Makino and N. Itoh, Tetrahedron: Asymmetry, 2005, 16, 2539-2549.

7 A. Li, L. Ye, F. Guo, X. Yang and H. Yu, J. Mol. Catal. B: Enzym., 2015,117, 31-37.

8 K. Honda, T. Ishige, M. Kataoka and S. Shimizu in Biocatalysis in the pharmaceutical and biotechnology industries, ed. R. N. Patel, Taylor and Francis, New York, 2006, pp. 529546.

9 U. Karl and A. Simon, Chimica Oggi - Chemistry Today. 2009, 27,1-4

10 B. H. Hoff and E. Sundby, Bioorg. Chem., 2013, 51, 31-47

11 D. Giacomini, P. Galletti, A. Quintavalla, G. Gucciardo and F. Paradisi, Chem. Commun. (Camb), 2007, 39, 4038-4040

12 I. Karume, M. M. Musa, O. Bsharat, M. Takahashi, S. M. Hamdan and B. E. Ali RSC Adv., 2016, 6, 96616-96622.

13 C. V. Voss, C. C. Gruber, K. Faber, T. Knaus, P. Macheroux and Kroutil W, J. Am. Chem. Soc., 2008, 130, 13969-13972. 
14 I. Karume, M. Takahashi, S. M. Hamdan and M. M. Musa, ChemCatChem, 2016, 8, 14591463.

15 M. M. Musa, J. M. Patel, C. M. Nealon, C. S. Kim, R. S. Phillips, I. Karume, J. Mol. Catal. B: Enzym., 2015, 115, 155-159.

16 C. C. Gruber, B. M. Nestl, J. Gross, P. Hildebrant, U. T. Bornscheuer, K. Faber and W. Kroutil, Chem.-Eur. J., 2007, 13, 8271-8276.

17 C. M. Nealon, T. P. Welsh, C. S. Kim and R. S. Phillips, Arch Biochem Biophys. 2016, 606, 151-156

18 Z. Sun, G. Li, A. Ilie and M. T. Reetz, Tetrahedron Lett., 2016, 57, 3648-3651.

19 M. L. Contente, I. Serra, L. Palazzolo, C. Parravicini, E. Gianazza, I. Eberini, A. Pinto, B. Guidi, F. Molinari and D. Romano, Org. Biomol. Chem., 2016, 14, 3404-3408.

20 A. Dudzik, W. Snoch, P. Borowiecki, J. Opalinska-Piskorz, M. Witko, J. Heider, and M. Szalenie, Appl. Microbiol. Biotechnol., 2015, 99, 5055-5069

21 C. Rodriguez, W. Borzecka, J. H. Sattler, W. Kroutil, I. Lavandera and V. Gotor, Org. Biomol. Chem., 2014, 12, 673-681

22 I. Lavandera, A. Kern, B. Ferreira-Silva, A. Glieder, D. S. Wildeman and W. Kroutil, J. Org. Chem., 2008, 73, 6003-6005.

23 J. Rocha-Martín, D. Vega, J. M. Bolivar, A. Hidalgo, J. Berenguer, J. M. Guisán and F. López-Gallego, Bioresour. Technol., 2012, 103, 343-350.

24 D. Zhu, B. A. Hyatt and L. Hua, J. Mol. Catal. B: Enzym., 2009, 56, 272-276

25 D. Alsafadi and F. Paradisi, Extremophiles, 2013, 17, 115-122

26 L. Olofsson, I. A. Nicholls and S. Wikman, Org. Biomol. Chem., 2005, 3, 750-75

27 L. M. Timpson, A. K. Liliensiek, D. Alsafadi, J. Cassidy, M. A. Sharkey, S. Liddell, T. Allers and F. Paradisi, Appl. Microbiol. Biotechnol., 2013, 97, 195-203.

28 L. M. Timpson, D. Alsafadi, C. Mac Donnchadha, S. Liddell, M. A. Sharkey and F. Paradisi, Extremophiles, 2012, 66, 57-66.

29 A. K. Liliensiek, J. Cassidy, G. Gucciardo, C. Whitely and F. Paradisi, Mol. Biotechnol., 2013, 55, 143-149.

30 D. Alsafadi and F. Paradisi, Mol. Biotechnol., 2014, 56, 240-247.

31 Z, Tan, H, Ma, Q, Li, L, Pu, Y, Cao, X, Qu, C, Zhu and H, Ying, Enzyme. Microb. Technol., 2016, 93-94, 191-199.

32 H. G. Naik, B. Yeniad, C. E. Koning and A. Heise, Org. Biomol. Chem., 2012, 10, 4961-4967.

33 A. Li, L. Ye, X. Yang, C. Yang, J. Gu and H. Yu, Chem. Commun., 2016, 52, 6284-6287.

34 E. B. Kurbanoglu, K. Zilbeyaz, M. Taskin and N.I Kurbanoglu, Tetrahedron: Asymmetry, 2009, 20, 2759-2763.

35 Z. Sun, R. Lonsdale, A. Ilie, G. Li, J. Zhou and M. T. Reetz, ACS Catal., 2016, 6, 1598-1605.

36 H. Neuvonen, K. Neuvonen, J. Chem. Soc. Perkin Trans. 2., 1999, 1497- 1502. 